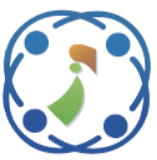

\title{
Fuzzy Logic Control for NLOS Identification Method in an Indoor Environment Using UWB Technology
}

\author{
Abbas Albaidhani ${ }^{1 *}$ \\ Ahlam Alsudani² \\ ${ }^{1}$ Technical Department-Ministry of Transport, Baghdad, Iraq \\ ${ }^{2}$ Department of Physics, College of Education, University of Baghdad, Baghdad, Iraq \\ * Corresponding author's Email: abbassabbar.ghali@e-campus.uab.cat
}

\begin{abstract}
For location-based applications, wireless systems in an indoor environment often operate under non-lineof-sight (NLOS)conditions that may cause ranging errors. A promising technology for location-aware sensor networks is an Ultra-wide bandwidth (UWB) transmission due to its robust operation in harsh environments, fine delay resolution, and power efficiency. However, the existence of walls and other obstacles causes a notable challenge in terms of localization, as they can result in positively biased distance estimates. A non-line-of-sight (NLOS) identification and mitigation technique created for UWB technology. A distance mitigation method is proposed using online line-of-sight (LOS) and NLOS identification by Fuzzy logic control decision. The result of the proposed method shows $99 \%$ of the accuracy of the proposed NLOS identification method and less than $20 \mathrm{~cm}$ of the measured distance error.
\end{abstract}

Keywords: WSN, Indoor positioning systems, UWB technology, LOS, NLOS, Fuzzy logic control.

\section{Introduction}

Nowadays, Indoor positioning sensing systems (IPs) are very popular and important in different places such as hospitals, airports, malls, factories, and etc. IPs determine the position of an object in a physical space continuously and in a real-time. Five major quality metrics of IPs exist: (1) precision and accuracy of the system; (2) coverage and its resolution; (3) latency in making location updates; (4) buildings infrastructure impact; and (5) random errors impacts on the system such as errors caused by signal interference and reflection [1]. To achieve the quality metrics, we should obtain a technology with a highly accepted ranging accuracy for indoor positioning applications. Different technologies in the market try to provide an accepted ranging accuracy. The accepted ranging accuracy depends on the indoor positioning application type. Some applications acquire centimeter positioning accuracy, and others may need for one-meter level accuracy such as applications in industrial metrology as well as for robot-and pedestrian navigation [2].
Indoor localization is an emerging technology that demands a theoretical and analytic background. The authors of [3] realize the necessity for the essential study of the characterization of indoor radio propagation and its effect on the accuracy of such systems. System design and performance evaluation need a framework for the growth and success of this technology. Four areas of challenges to locate a mobile position in an environment which are cost and complexity, performance, security, and application requirements are identified by [4].

In this work, we are interested in the performance challenge because it is corresponding to the positioning accuracy, and we explain it briefly in the text below.

The most important performance metric is the accuracy of the position information. Normally, this reported as an error distance between the estimated location and the actual mobile location. The report of accuracy should include the confidence interval or percentage of successful location detection which is called the location precision. Indoor environments distribute as structured or known, semi - structured 
and unstructured or unknown depending on the control that the IPs possesses over them $[5,6]$.

In a radio frequency (RF) communication network, Localization distribute into range-free and range-based techniques [7], where the range-free approach is radio signal strength indication (RSSI). A model of theoretical or experimental of the propagation of a signal in this approach is translated into position or distance estimations [8, 9]. The range based approach according to distance measurements between transceivers using the time of arrival (TOA), time difference of arrival(TDOA) or tow way ranging time of flight (TWRTOF) [10].

UWB is considered one of the most precise approaches of the aforementioned forms of RF technology because it ables to present positioning estimate with centimeter-level accuracy $[11,12]$. It widely implanted for ranging estimation and building an indoor positioning system. In the indoor environment, the propagation channels divided into LOS and NLOS. The NLOS divided into soft NLOS and hard NLOS based on the radio signal attenuation. In the UWB signals, very short pulses transmitted and detected permitting for high accuracy of positioning because of the precise computation of signal delays. In an indoor environment, a length of propagation path is not always a suitable indication of the ranging between a sender and receiver. Therefore, these systems are predominately limited to the LOS conditions [13]. It accurately measures the ranging in the LOS channel but suffers in the NLOS channel, and the error in the estimated distance is considerably high $[14,15]$ which effects the positioning accuracy. The goal of this work is to identify the NLOS channels and divide them into hard and soft to facilitate the mitigation method for obtaining a precise distance measurement.

In this paper, we present a novel algorithm to identify the NLOS and LOS propagation channels that affect the UWB signal using a Fuzzy logic control decision, then mitigate the error in distance measurement to obtain a precise ranging measurement. In this work, the error of the distance measurement achieved an average of less than 20 $\mathrm{cm}$ in the NLOS (hard and soft) channels and less than $5 \mathrm{~cm}$ in the LOS channel.

The organization of the rest of this paper as follows: a literature survey related to this work presents in Section 2. Section 3 represents the problem formulation related to the IPs. Section 4 presents the proposed framework (NLOS and LOS identification and mitigation method). Section 5 presents the experimental activities implemented in this work. Result and discussion offer in section 6 . Finally, a conclusion presents in section 7 .

\section{Literature survey}

In this section, we present some of the LOS and NLOS identification and mitigation methods related to the proposed work created in the last years which used the received signal power. In the proposed work, we take into consideration three parameters extracted from the UWB signal used for NLOS identification and mitigation. These parameters are i) the total received signal power (RSP); ii) the power of the signals corresponding to the first path (FSP), and iii) the signal to noise ratio (SNR).

Recently, IH Brahmi presented an indoor positioning system based on a fuzzy logic approach called Fuzzy-LSE (FLSE) [16]. The authors, however, centered the derivation of the Fuzzy logic scheme to cope only with the localization phase. To do so, the Fuzzy approach based on considering four parameters (power, LOS value, Noise, and distance) to derive the indoor positioning system. It is also worth noting that NLOS identification in that work based on the identification method created by Decawave company [21]. As shown in [14], this methodology has two main negative drawbacks. First, the NLOS identification method does not consider all the possible combination of received signal power (RSP) - first path signal power(FSP) differences Secondly, the estimated received power by (UWB) device differs from the actual values above $-85 \mathrm{dBm}$. Both problems solved by our methodology.

In 2018, Dae-Ho Kim [17] presented another method to identify the NLOS and LOS channels in a different indoor environment. The author enhanced the technique used in [14] by adding different types of the direct path of the UWB signal to experimentally evaluate it if it comes through a LOS or NLOS (hard and soft) channel. In this work, only an NLOS and LOS identification method created. The author of [18], 2018, presented an algorithm to identify the LOS and NLOS channels and improve the positioning accuracy using the UWB technology implementing decawave (DW) 1000 device. In this work, the author computed the received signal power (RSP) based on the estimated distance extracted from the DW 1000 devise. Then, they identified the difference between RSP and FSP. If it is less than $6 \mathrm{~dB}$ the propagation channel be LOS, and else, the propagation channel is NLOS. Then, the RSP is used to compute the path loss component used to measure the signal-to-noise ratio (SNR) 
implemented to remove the anchor nodes having less SNR when creating a positioning system.

The authors of [14], 2016, presented an NLOS and LOS identification and mitigation method using a commercial UWB device. The authors experimentally evaluated and validated (UWB) device called (DW 1000), evaluation kit 1000 (EVK 1000), on a walking human in an indoor environment of LOS and NLOS channels, and then created an algorithm to identify and mitigate the NLOS channels. In this work, they identified the difference between RSP and FSP. If it is less than 6 $\mathrm{dB}$, the channel is more likely to be a LOS, and if it ranges between 6-10 $\mathrm{dB}$ and the instant RSP is greater than the average RSP $(-87 \mathrm{dBm})$, the channel is more likely to be NLOS or else, it will be LOS. And if it is greater than $10 \mathrm{~dB}$, the channel is more likely considered an NLOS. The ranging error mitigation process is done by using some extra information related to the maps of the environment held the experiment. The mitigation method measured distance with an error which about $60 \mathrm{~cm}$ in the hard-NLOS channel.

In 2015, Bo You [19] offered an NLOS identification and mitigation method depending on the RSP and FSP. He stated two different thresholds ( $\alpha$ and $\beta$ ) where $\alpha$ and $\beta$ are $25 \%$ and $10 \%$ respectively. These thresholds are according to DW1000 user manual [21]. Then, the authors defined a ratio between the FSP and RSP. The channel is (LOS) if the ratio is higher than the threshold $(\alpha)$, and it is (NLOS) if the ratio is lower than threshold $\beta$. For an NLOS ranging error mitigation, the authors obtained the estimated ranging error experimentally and removed it from the estimated distance to obtain the mitigated distance. In this work, the authors did not mention what is the ranging accuracy after the proposed method, but they only mentioned the localization accuracy had been increased $50 \%$ when using the traditional least square localization method with the proposed method than without it.

The authors of [20] implemented an algorithm to identify the propagation channel and enhance the ranging accuracy. In this method, the received signal strength (RSS) measurements from the Wi-Fi signal. The authors exploited different statistical properties of the RSS time series and used two approaches based on machine learning and a third depending on hypothesis testing to separate LOS-NLOS measurements. This algorithm of NLOS identification and mitigation conducted implementing only RSS from real experiments with mobile devices. The authors claimed that the created algorithm is able to distinguish between LOS and
NLOS conditions with an accuracy of around 95\%. Furthermore, the presented approach improves the accuracy of the estimated distance by $60 \%$ as compared to previous NLOS mitigation approaches, and they acquired an enhancement in distance estimation accuracy of $50 \%$.

From the literature aforementioned above and other previous literature that used the received power of (UWB) signal to create an indoor positioning system or NLOS identification method, we should mention the main drawback of these works which is the value of the received power when it estimated by (UWB) device. This value diverges from its true value when the received power is above $-85 \mathrm{dBm}$ (see Fig. 1), which results in a significant error in the positioning or ranging accuracy [3]. In this paper, we overcome the aforementioned problem, and so we can conclude that our paper will improve the ranging accuracy in different indoor environments and situations when the ranging accuracy for pedestrians enhanced with less than $20 \mathrm{~cm}$ error.

\section{Problem formulation}

As we mentioned in the literature survey section about the main drawback of the created systems mentioned above, we overcome this drawback using the fuzzy logic (FL) and therefore, the accuracy of (NLOS) identification method is highly increased comparing with the other methods. Also, the created databases (Tables 2 and 4) help us to implement the mitigation process without the need for extra information about the environment such us maps and enhance the estimated distance achieving a distance accuracy of less than $20 \mathrm{~cm}$ error which is much better than the accuracy achieved by the other studies. In this section, we described the problem of (NLOS) identification and mitigation method and solved implementing the proposed method. We installed two UWB sensors (transmitter TX and receiver RX) in three different scenarios as depicted in Fig. 2. The first scenario presents the LOS channel when the signal travels between the sensors with a direct path in free space without any obstruction. The second scenario presents the soft NLOS channel when the signal travels between the sensors with a direct path obstructed by one wall of $30 \mathrm{~cm}$ thickness. The last scenario presents the hard NLOS when the signal travels between the sensors with a direct path obstructed by two walls of $30 \mathrm{~cm}$ thickness of each wall.

In our method, first, we identify the propagation channel as LOS, hard NLOS, and soft NLOS using the Fuzzy logic (FL) method. The input parameters 
used in the FL are RSP, FSP, and SNR extracted from (UWB) sensor and therefore, the last step of the proposed method is the mitigation process (distance enhancement) implementing the databases shown in Tables 3 and 2 to compute the enhanced distance using Eq. (3) as shown in the proposed framework section.

\section{Proposed framework}

In this section, we present briefly the steps of the proposed algorithm which consists of the NLOS identification and mitigation process of different propagation channels in an indoor environment.

\subsection{NLOS identification method}

1) The main parameters:

The NLOS identification method depends on three parameters; i) estimated power for just the first path signal (the signal power in the first path FSP), ii) estimated receive power figure (the received signal power RSP), and iii) signal to noise ratio (SNR), and they explained below. These parameters are extracted in this work from the implemented UWB device (DW 1000-EVK 1000).

a. According to [21] and by using Eq. (1), signal power in the first path FSP computed

$$
F S P(\mathrm{~dB} m)=10 \times \log _{10}\left(\frac{F 1^{2}+F 2^{2}+F 3^{2}}{N^{2}}\right)-A
$$

where, $F 1 ; F 2$, and $F 3$ denote the First Path Amplitude (point 1), the First Path Amplitude (point 2), and the First Path Amplitude (point 3) respectively. All points mentioned above are measured by the DW 1000 .

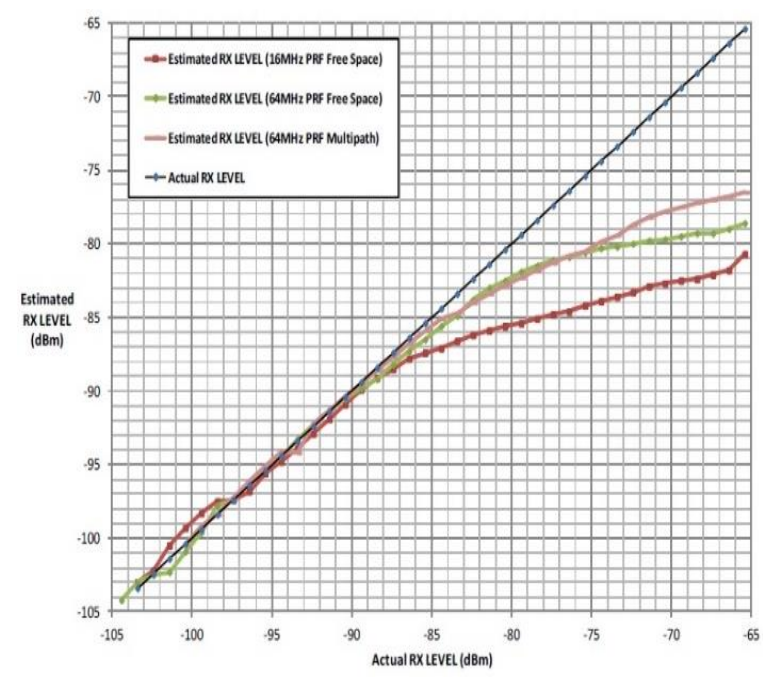

Figure. 1 Estimated RX level versus true RX level [21]

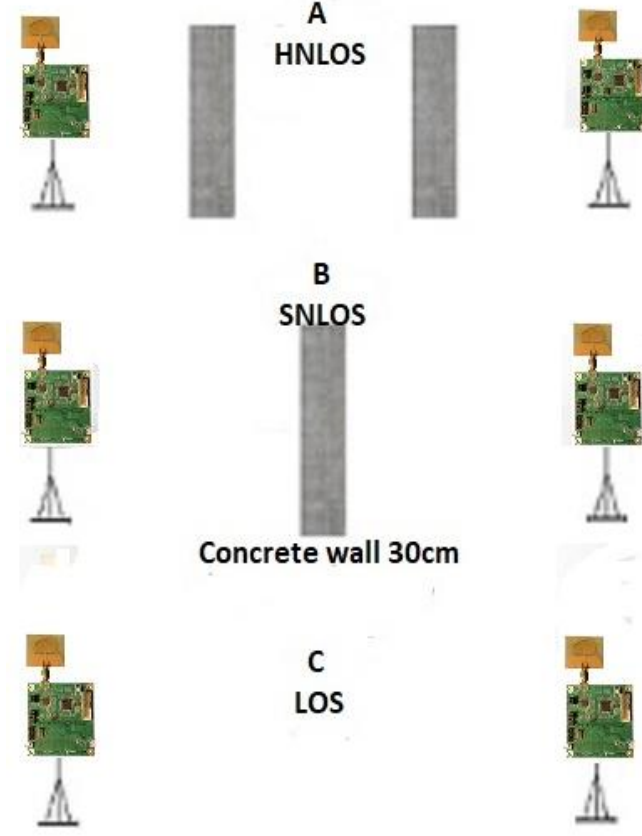

Figure. 2 The proposed scenario

b. The received signal power $R S P$ is computed using Eq. (2) according to [21].

$$
R S P(d B m)=10 \log _{10}\left(\frac{2^{17} c}{N^{2}}\right)-A
$$

Where, $C, A$, and $N$ denote the Channel Impulse Response Power value (CIR), constant 113.77 for Pulse repetition frequency (PRF) of $16 \mathrm{MHz}$, or, the constant 121.74 for PRF of $64 \mathrm{MHz}$, and the Preamble Accumulation Count value respectively.

c. Signal to noise ratio (SNR $(\mathrm{dB}))$ is computed by the DW 1000-EVK 1000 device.

All parameters mentioned in the items $\mathrm{a}, \mathrm{b}$, and $\mathrm{c}$ above implemented in this work are extracted from the UWB device (DW1000-EVK 1000) implemented in this method.

\section{2) Fuzzy logic control:}

In the NLOS identification method and after obtaining the main parameters explained above, the main step is the Fuzzy logic control technique. So, first, we present below an overview of the concept of the Fuzzy logic control Fuzzy logic is an approach based on several logic values rather than binary logic (two logic values) Two valued logic overwhelmingly, considers 1 to be true and 0 to be false. However, fuzzy logic transacts with truth values between 0 and 1 , and these values consider as intensity (degrees) of truth. We apply the following 
three steps to use Fuzzy logic in a real application [22]:

1. Fuzzification: In this step, the classical data converted into Membership Functions (MFs) or fuzzy data.

2. Fuzzy Inference Process: Control rules are combined with the output of the previous step (membership Functions) to derive the fuzzy output in this step.

3. Defuzzfication: By implementing different methods, each corresponding output is computed and put them into a table: the lookup table. Pick up the output from the lookup table according to the current input during an application. The three steps above are explained briefly in the next section (experimental activities).

\subsection{NLOS mitigation (ranging enhancement)}

After identifying the appropriate propagation channel from the NLOS identification method, the ranging enhancement technique to obtain the best possible ranging accuracy is implemented using Eq. (3) below. More details regarding the distance enhancement method explained in the experimental activities section.

$$
d=\hat{d}-\text { Bias }_{a v r}
$$

Where, $d, \widehat{d}$, Bias $_{a v r}$ denote the enhanced distance after the NLOS identification method, distance extracted from the DW1000-EVK 1000, and the average of the Bias distance computed as explained in the experimental activities section respectively. The average value of the distance bias used because, in every propagation channel, the variance of the estimated distance is close to zero mean as we have shown in our work, anchor selection for UWB indoor positioning [23] and therefore, the average value will be more suitable than the median value. We should mention that the device implemented in this experiment has a positive Bias in the LOS channel [24] and therefore the Bias is also positive in the NLOS channel. All steps of the proposed framework are explained in the algorithm I.

\begin{tabular}{l}
\hline $\begin{array}{l}\text { Algorithm 1 NLOS } \\
\text { mitigation method }\end{array}$ \\
\hline 1. procedure EXTRACT INFORMATION \\
\hline
\end{tabular}

\section{FSP, RSP, SNR \\ Extracted \\ from the \\ EVK 1000}

\section{3. end procedure}

\section{1. procedure NLOS IDENTIFICATION METHOD}

2. implement Fuzzy logic control

3. range of Fuzzy input set

4. Fuzzy control rule

5. range of the Fuzzy output set

$6 . \quad$ defuzzyfication method

$>$ centre of gravity (COG)

$$
P=\frac{\sum_{i=1}^{n} A_{i \times} c_{i}}{\sum_{i=1}^{n} A_{i}}
$$

\section{7. end procedure}

1. procedure NLOS MITIGATION.

- Distance enhancement

2. create a database of the average of the extracted information

3. create a database of the Bias for every propagation channel

4. Find the enhanced distance

5. end procedure

$$
d=\hat{d}-\text { Bias }_{\text {avr }}
$$

\section{Fuzzy logic-based NLOS}

\subsection{Identification and mitigation}

We experimented (3) different propagation channels in this work shown in Fig. 2 and explained below: We placed Two EVK 1000 as follows:

(1) LOS channel: The traveling signal (UWB signal) passes between two wireless sensors (DW 1000-EVK 1000) through a direct path without any restriction.

(2) Soft NLOS channel: The signal travels through a $30 \mathrm{~cm}$ thickness wall.

(3) Hard NLOS channel: The signal travels through two $30 \mathrm{~cm}$ thickness walls.

- $\quad$ Propagation distances range between $2 m$ and $22 \mathrm{~m}$. The first Fresnel zone in the vicinity of TX and RX is not blocked.

- Measuring the estimated distance, the RSP, FSP, and SNR for 1000 times to create the database depicted in Table 4. 
Next, we introduce the Fuzzy Logic (FL)-based strategy to find out a distance correction Bias $_{\text {avr }}$ from the input data $R S P, F S P$ and $S N R$.

\subsection{NLOS identification}

The goal in our Fuzzy Logic approach is to output a score $\mathrm{P}$ that helps in identifying the type of channel as depicted in Table 2. We consider three input values: $S N R, R S P$ and $d i f=F S P-R S P$.
.i) $R S P=[-98 \mathrm{dBm}-78 \mathrm{dBm}]$,
ii) $S N R=[-18 d B 1 d B]$, and
iii) dif $=\left[\begin{array}{llll}-25 & d B & 1 & d B\end{array}\right]$.

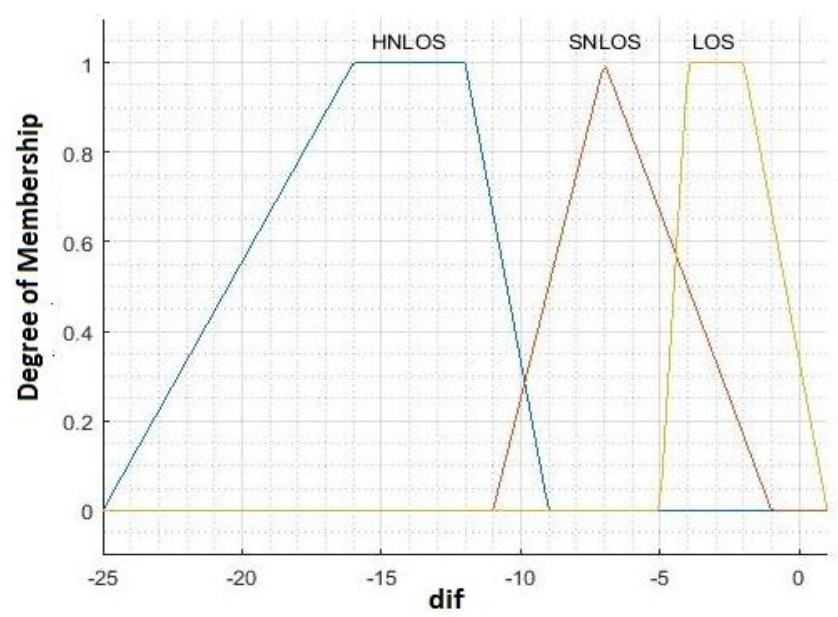

Figure. 3 The degree of the membership function of the dif (input) parameter

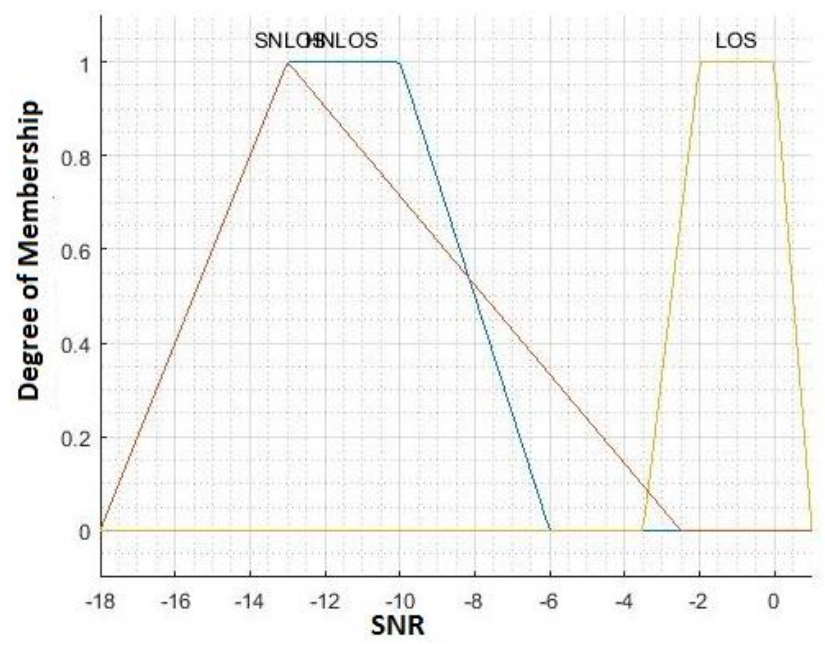

Figure. 4 The degree of the membership function of the SNR (input) parameter
Table 1. Fuzzy IF-THEN rules

\begin{tabular}{|c|c|c|c|c|}
\hline Rule & RSP & dif & SNR & $\mathrm{P}$ \\
\hline 1 & Low & Low & Low & Very Low \\
\hline 2 & Low & Low & Medium & Low \\
\hline 3 & Low & Low & High & Rather Low \\
\hline 4 & Low & Medium & Low & $\begin{array}{l}\text { Rather } \\
\text { Medium }\end{array}$ \\
\hline 5 & Low & Medium & Medium & Medium \\
\hline 6 & Low & Medium & High & $\begin{array}{l}\text { Very } \\
\text { medium }\end{array}$ \\
\hline 7 & Low & High & Low & $\begin{array}{l}\text { Rather } \\
\text { Medium }\end{array}$ \\
\hline 8 & Low & High & Medium & Medium \\
\hline 9 & Low & High & High & $\begin{array}{l}\text { Very } \\
\text { Medium }\end{array}$ \\
\hline 10 & Medium & Low & Low & Rather Low \\
\hline 11 & Medium & Low & Medium & $\begin{array}{l}\text { Low } \\
\text { Medium }\end{array}$ \\
\hline 12 & Medium & Low & High & $\begin{array}{l}\text { Rather } \\
\text { Medium }\end{array}$ \\
\hline 13 & Medium & Medium & Low & $\begin{array}{l}\text { Rather } \\
\text { Medium }\end{array}$ \\
\hline 14 & Medium & Medium & Medium & Medium \\
\hline 15 & Medium & Medium & High & $\begin{array}{l}\text { Very } \\
\text { Medium }\end{array}$ \\
\hline 16 & Medium & High & Low & Medium \\
\hline 17 & Medium & High & Medium & $\begin{array}{l}\text { Very } \\
\text { Medium }\end{array}$ \\
\hline 18 & Medium & High & High & High \\
\hline 19 & High & Low & Low & $\begin{array}{l}\text { Rather } \\
\text { Medium }\end{array}$ \\
\hline 20 & High & Low & Medium & Medium \\
\hline 21 & High & Low & High & $\begin{array}{l}\text { Rather } \\
\text { High }\end{array}$ \\
\hline 22 & High & Medium & Low & Medium \\
\hline 23 & High & Medium & Medium & $\begin{array}{l}\text { Very } \\
\text { Medium }\end{array}$ \\
\hline 24 & High & Medium & High & High \\
\hline 25 & High & High & Low & $\begin{array}{l}\text { Rather } \\
\text { High }\end{array}$ \\
\hline 26 & High & High & Medium & High \\
\hline 27 & High & High & High & Very High \\
\hline
\end{tabular}


Table 2. Type of the propagation channel corresponding to the range of $\mathrm{P}$ bias value

\begin{tabular}{|l|l|l|}
\hline $\begin{array}{l}\text { Range } \\
\text { of P }\end{array}$ & channel type & Bias value $m$ \\
\hline $25-35$ & $\begin{array}{l}\text { Very hard } \\
\text { NLOS }\end{array}$ & 1.3 \\
\hline $36-39$ & hard NLOS & 0.8 \\
\hline $40-42$ & $\begin{array}{l}\text { Rather hard } \\
\text { NLOS }\end{array}$ & 0.5 \\
\hline $43-44$ & $\begin{array}{l}\text { Very soft } \\
\text { NLOS }\end{array}$ & 0.5 \\
\hline $45-51$ & $\begin{array}{l}\text { Soft NLOS } \\
\geq 56\end{array}$ & $\begin{array}{l}\text { Rather soft } \\
\text { NLOS }\end{array}$ \\
\hline $52-55$ & 0.15 \\
\hline
\end{tabular}

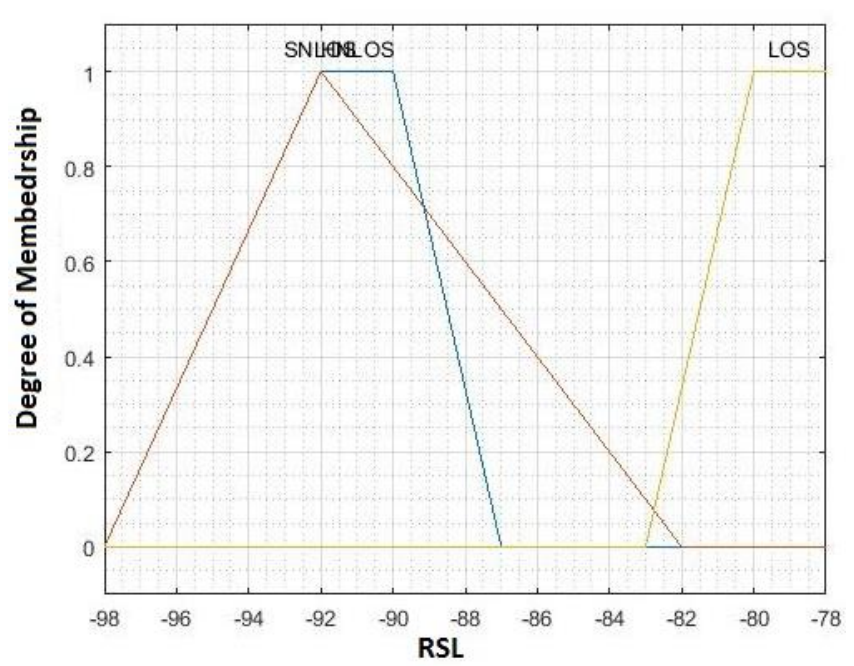

Figure. 5 The degree of the membership function of the RSL (input) parameter

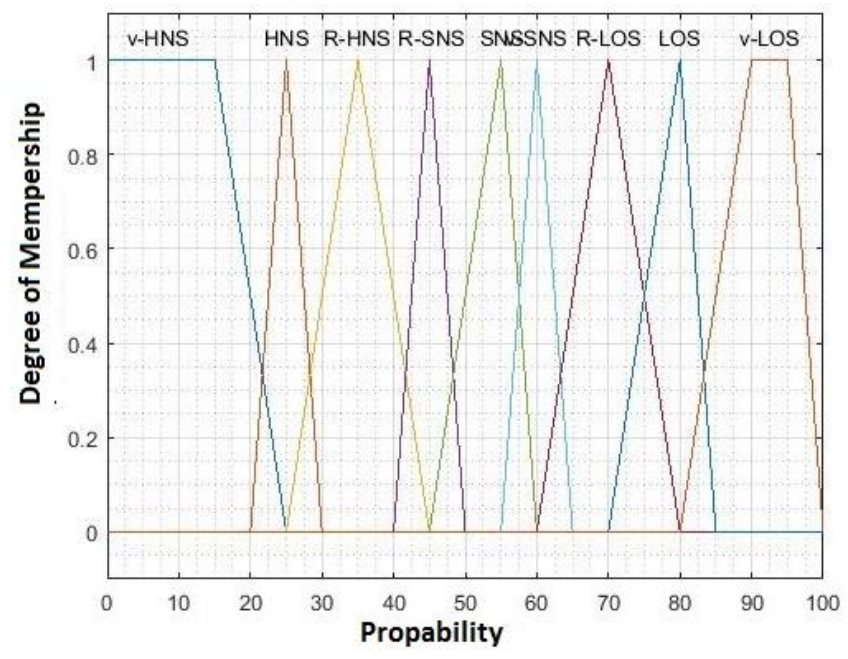

Figure. 6 The degree of the membership of the output sets
The input fuzzy sets for dif, SNR and RSP are depicted in Figs. 3, 4, and 5, respectively. The membership function types of the aforementioned inputs are trapezoidal and triangle. After the input fuzzification, the fuzzy rules applied to each input parameter are explained in the Fuzzy IF-THEN rules as shown in Table 1. The fuzzy rule applied in this approach is IF-THEN rule. All rules activate to some extent in a fuzzy logic technique or in different meaning they active partially. If the antecedent is correct to some degree of membership, then the consequent is correct to the same degree. A rule of Fuzzy may have multiple antecedence, for example Table 1: IF SNR has a value of high (for example between -5 and 1), RSP has a value of low, and FSP has a low value, Then the output of the FL (P) will be Rather Low which means close to the NLOS channel. The Fuzzy Logic rules were explained as in Table 1. The output set of FL is divided into different ranges of the propagation channel types as shown in Fig. 6.

The last step of the FL is the defuzzification process. The fuzzy system output $(\mathrm{P})$ is defuzzified implementing the center of gravity defuzzification method as expressed in Eq. (4). P is unit-less value, and we created a metric of its range. This metric is as follow: LOS: $\mathrm{P}>56$, hard NLOS: $\mathrm{P}=[30-41]$, and soft NLOS: $\mathrm{P}=[42-$ 57]. Figs. 7, 8, and 9 depict the output of the Fuzzy system (P) as shown below.

According to the $P$ metric (Eq. (4)), we will be able to identify the propagation channel into LOS or hard and soft NLOS. The horizontal coordinate in the figures aforementioned above presents the number of the points (index of the receiver) ranged between $2 m$ and $22 m$ tested in this experiment, and the vertical coordinate presents the output of the Fuzzy system $(P)$.

$$
P=\frac{\sum_{i=1}^{n} A_{i \times} c_{i}}{\sum_{i=1}^{n} A_{i}}
$$

Where, $P, A, c$, and $n=27$ denote the Fuzzy system output, the subarea of the input system, and the centroid of the subarea, and the total number of the subareas respectively. After the determination of the type of the propagation channel, the NLOS mitigation method (distance enhancement) is implemented as explained below.

\subsection{NLOS mitigation}

The idea of distance enhancement is to obtain the best possible ranging accuracy. To reach this goal, we experimentally created a database of the 
measured distance in different types of propagation channels (LOS, soft NLOS, and hard NLOS).This database also includes the average Bias, RSP, FSP, and SNR of the measured distance computed for every channel as shown in Table 4, and therefore, we divide the aforementioned channels into three probabilities, rather (low), medium, and very(high) as shown in Table 2, then, using the information available in Tables 2 and 4 (see Appendix I) and applying the Eq. (3) to obtain the final result of the enhanced measured distance.

\section{Result and discussion}

In this section, we present the results of the proposed NLOS identification and mitigation approach of the UWB signal in an indoor environment obtained experimentally using the FL. As we mentioned in section IV, the propagation channel in this experiment is divided into three types. The first type is called a LOS channel, where the travelled signal passes through free space with no obstructions. A soft NLOS channel is the second type, where the travelled signal passes through one concrete wall of $30 \mathrm{~cm}$ width. The third type is called a hard NLOS, where the travelled signal passes through two concrete walls of $60 \mathrm{~cm}$ total width. After obtaining the result of the NLOS identification method, we tested the sensitivity and specificity metrics to assess the accuracy of the proposed NLOS identification method. Table 3 shows the identification accuracy of the proposed method and the methods created by $[14,19]$. The two, Eqs. (5) and (6), below express the true positive rate (TPR), sensitivity, recall, and true negative rate (TNR) and specificity (SPC), respectively.

Table 3. Accuracy of NLOS identification algorithm compared to the methods crated by $[14,19]$

\begin{tabular}{|c|c|c|c|c|c|}
\hline $\begin{array}{c}\text { Channel } \\
\text { mode }\end{array}$ & $\begin{array}{c}\text { distances } \\
(\mathrm{m})\end{array}$ & TPR & TNR & ACC & Note \\
\hline 2 & $4-20$ & $0: 897$ & $0: 983$ & $0: 929$ & $\begin{array}{c}{[14]} \\
\text { algorithm }\end{array}$ \\
\hline 2 & $4-20$ & $0: 761$ & $0: 891$ & $0: 826$ & $\begin{array}{c}{[19]} \\
\text { algorithm }\end{array}$ \\
\hline 2 & $4-20$ & $0: 989$ & $0: 998$ & $0: 990$ & $\begin{array}{c}\text { Proposed } \\
\text { algorithm }\end{array}$ \\
\hline
\end{tabular}

$$
\begin{aligned}
& T P R=\frac{\sum \text { True Positive }}{\sum \text { Condition Positive }} \\
& T N R=\frac{\sum \text { True Negative }}{\sum \text { Condition Negative }}
\end{aligned}
$$

And the accuracy equation (ACC) shown in Eq. (7) is

$$
A C C=\frac{\sum \text { True Positive }+ \text { True Neqative }}{\sum \text { total Population }}
$$

Then, we implemented the relative error (average of 1000 times) expressed in Eq. (8) and the average error of the enhanced distance to assess the ranging accuracy.

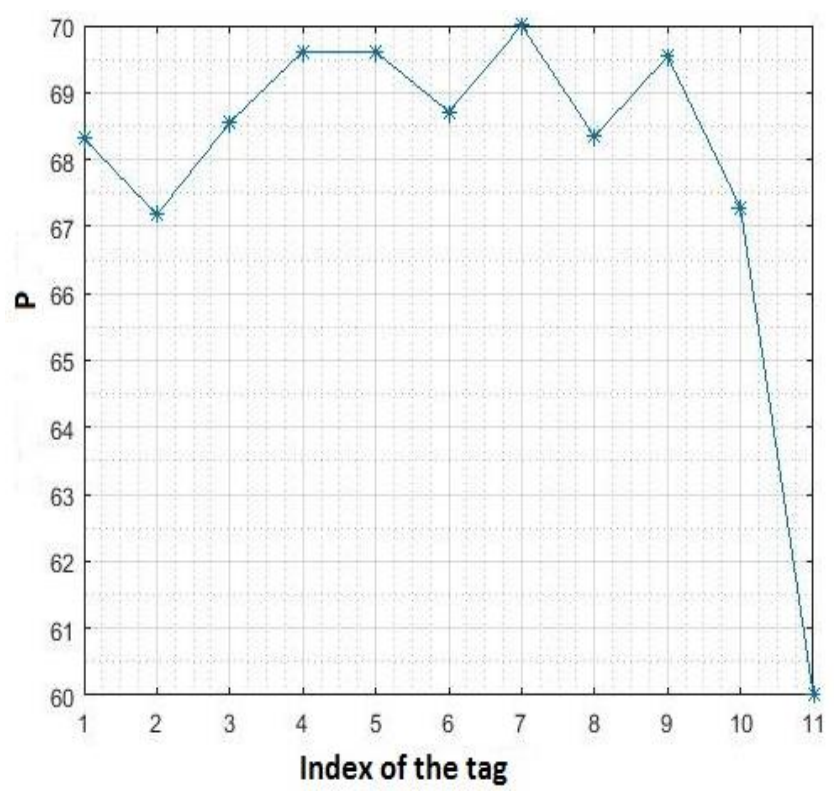

Figure. 7 The output of the Fuzzy system (P) for LOS

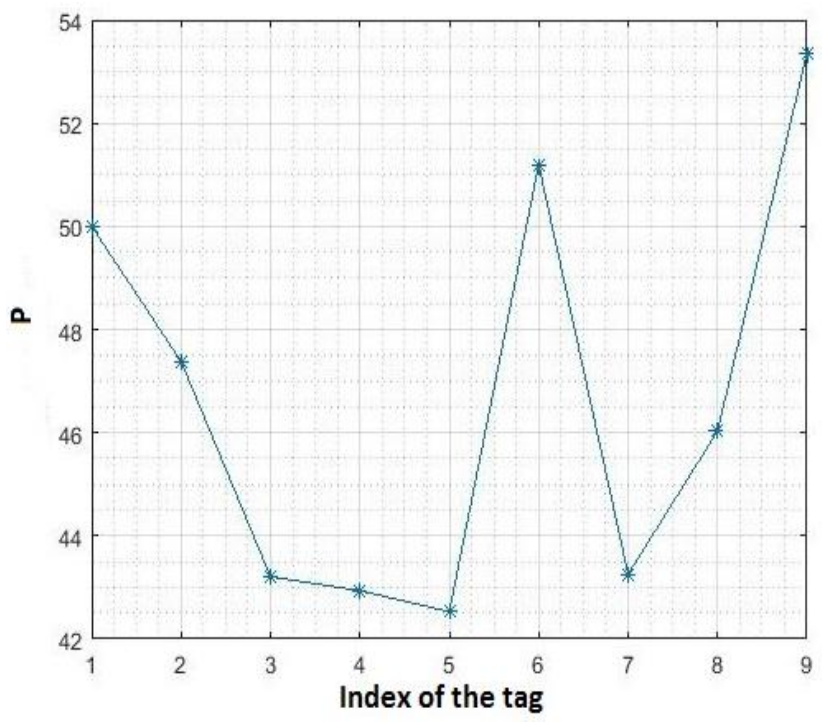

Figure. 8 The output of the Fuzzy system (P) for soft NLOS channel 


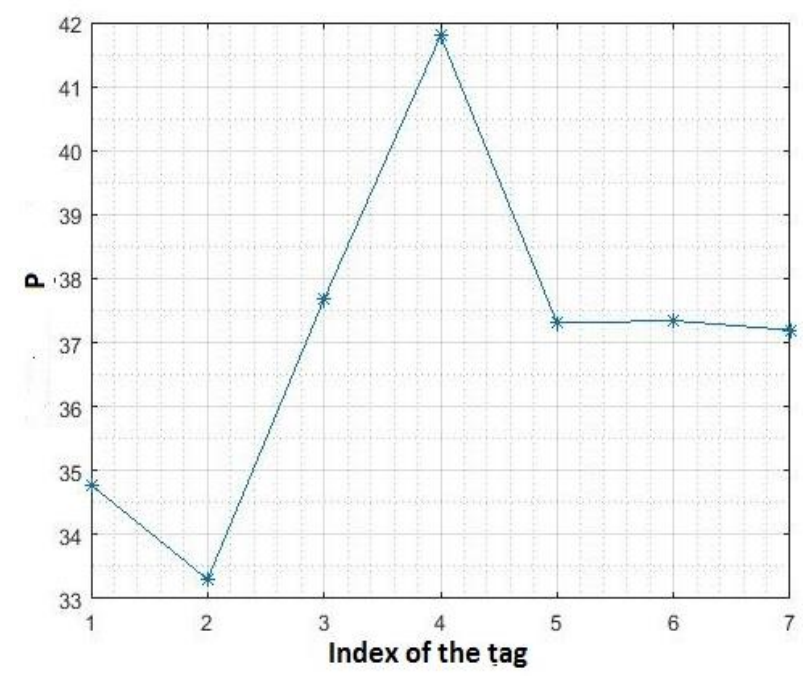

Figure. 9 The output of the Fuzzy system (P) for hard NLOS channel

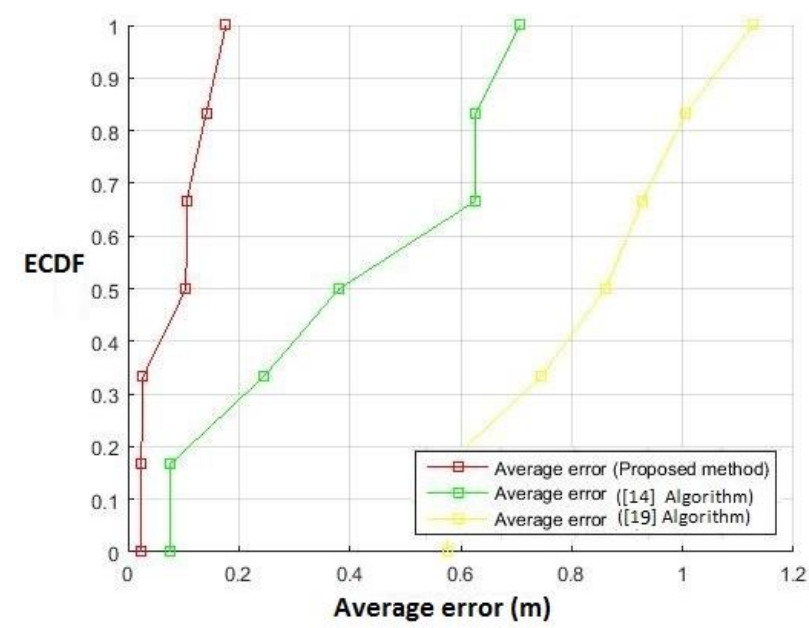

Figure. 10 The average of ranging error in a hard NLOS channel for the proposed method (red curve) and the methods created by [14] (green curve) and by [19] (yellow curve)

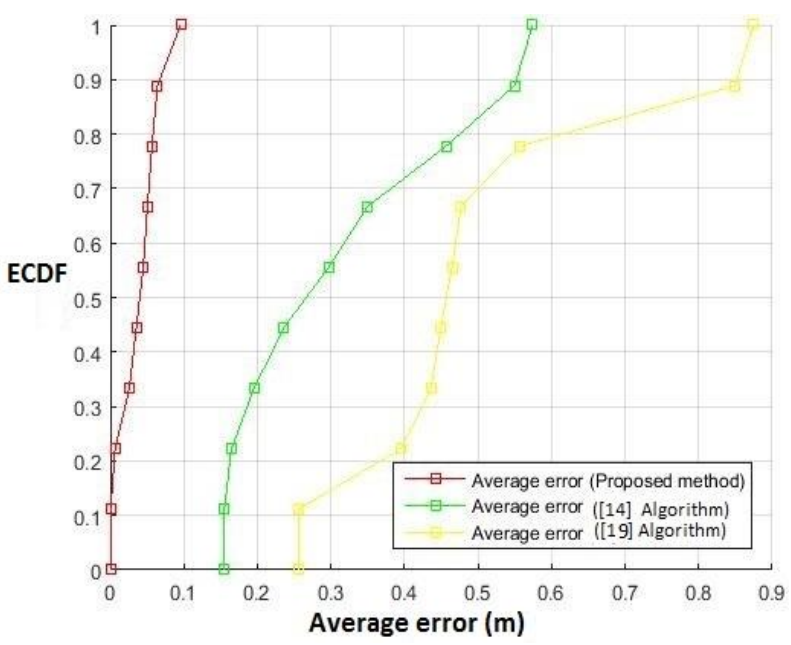

Figure. 11 The average of ranging error in a soft NLOS channel for the proposed method (red curve) and the methods created by [14] (green curve) and by [19] (yellow curve)

$$
E_{r}=\frac{d_{i(n)-r}}{r} \times 100 \%
$$

where, $r, d$, and $n$ denote the real distance, enhanced distance, and the total number of the enhanced distance respectively.

For the NLOS mitigation process, we compute the average and relative error of the enhanced distance in hard and soft NLOS channels, and then compared the Empirical cumulative distributed function (ECDF) of the results with the ECDF of the results of the NLOS mitigation methods created by $[14,19]$ as shown in Figs. 10, 11, 12, and 13. Figs. 10 and 12 present the average of ranging error in a hard NLOS channel and it is clear from these figures that the distance error for the proposed algorithm is around $20 \mathrm{~cm}$. Figs. 11 and 13 present the rational error of the enhanced distance. For the proposed algorithm, it is clear from these figures the rational error is between 1 and $2 \%$.

Hint: The UWB signal will face a difficulty of propagation when traveling beyond $20 \mathrm{~m}$ as we have shown in $[14,23]$ in a hard NLOS channel and therefore, we test our method for 7 points with $2 \mathrm{~m}$ apart between each point up to $20 \mathrm{~m}$.

It is clear from the result of the proposed method that the proposed algorithm has $99 \%$ of an accuracy of NLOS identification approach and a relative error of the enhanced estimated distance approximately below $1.3 \%$ in $99 \%$ of the cases whereas the methods in $[14,19]$ have $92 \%$ and $82 \%$ of an accuracy of NLOS identification approach and relative error of the enhanced estimated distance around $8 \%$ and $14 \%$ respectively in $99 \%$ of the cases.

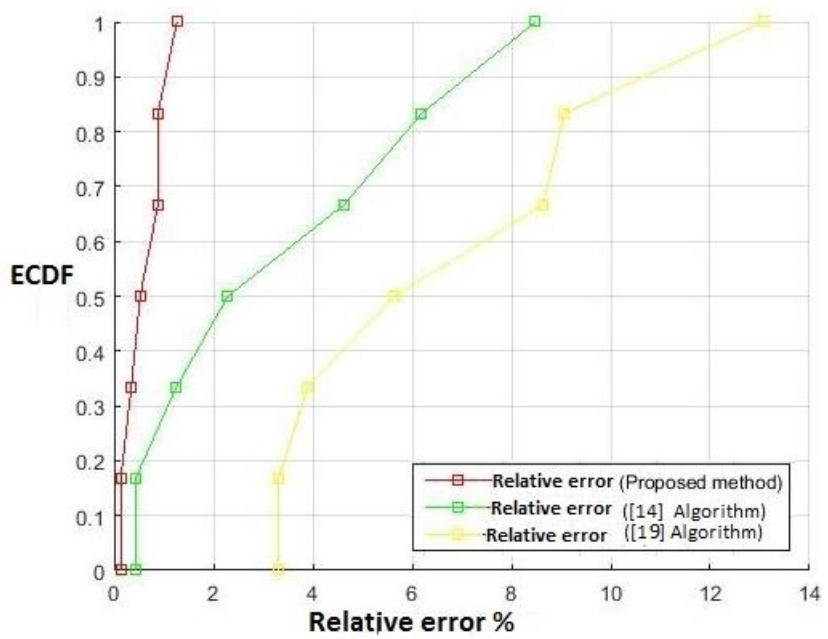

Figure. 12 The relative error of the enhanced and real distances in a hard NLOS channel for the proposed method (red curve) and the methods created by [14] (green curve) and by [19] (yellow curve) 


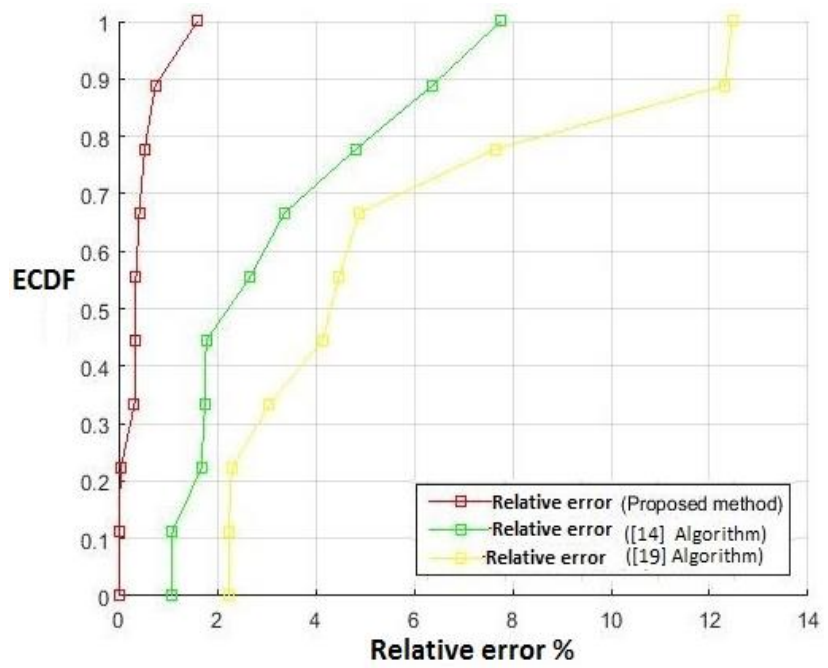

Figure. 13 The relative error of the enhanced and real distances in a soft NLOS channel for the proposed method (red curve) and the methods created by [14] (green curve) and by [19] (yellow curve)

\section{Conclusion}

In this work, a novel method is implemented to overcome the main limitation of UWB signal in a harsh indoor environment which is the ranging accuracy and to solve the main drawback that effects the ranging accuracy in the previous work aforementioned in the literature survey section. The identification and mitigation algorithm using Fuzzy logic control decision and the information extracted from the UWB commercial device is a confident method that provides a very high acceptable NLOS identification method about $99 \%$ and ranging accuracy with less than $20 \mathrm{~cm}$ of average error of the distance. In average, The NLOS identification method and the raging accuracy obtained in this work is higher among other identification and mitigation techniques in the market. The use of the proposed NLOS identification system in creating an indoor positioning system in a harsh environment is our goal in the future work.

\section{References}

[1] H. Liu and T. Kaiser, "Survey of Wireless Indoor Positioning techniques and Systems", IEEE, Transaction on Systems, Man, and Cybernetics, Vol. 37, No. 6, pp. 1067-1080, 2007.

[2] R. Mautz and S. Tilch, "Survey of Optical Indoor Positioning Systems", IEEE, IPIN Conference, pp. 1-7, 2011.

[3] K. Pahlavan, X. Li, and J. Makela, "Indoor geolocation science and technology", IEEE
Commun. Mag., Vol. 40, No. 2, pp. 112-118, 2002.

[4] P. Krishnamurthy, "Position location in mobile environments", In: Proc. NSF Workshop on Context-Aware Mobile Database Management $(C A M M)$, pp. 1-3, 2002.

[5] J. Callmer, "Autonomous localization in unknown environments", Linkoping University Electronic Press, Dissertations, No. 1520, ch 3, 2013.

[6] M. Algabri, H. Mathkour, H. Ramdane, and M. Alsulaiman, "Comparative study of soft computing techniques for mobile robot navigation in an unknown environment", Comput. Human Behav, Vol. 50, pp. 42-56, 2015.

[7] T. He, C. Huang, B. Blum, A. Stankovic, and T. Abdelzaher, "Range free localization schemes for large scale sensor networks", In: Proc of the 9th Annual International Conference on Mobile Computing and Networking, pp 81-95, 2003.

[8] P. Lourenco, P. Batista, P. Oliveira, C. Silvestre, and P. Chen, "A received signal strength indication-based localization system", In: Proc. of 21th Mediterranean Conference on Control Automation, pp. 1242-1247, 2013.

[9] L. Wu, H. Meng, Z. Lin, W. He, C. Peng, and H. Liang, "practical evaluation of radio signal strength for mobile robot localization", In: Proc of International Conference on Robotics and Biomimetics (ROBIO), pp. 516-522, 2009.

[10] Z. Farid, R. Nordin, and M. Ismail, "Recent advances in wireless indoor localization techniques and system", J. Comput. Netw. Commun., Vol. 2013, pp. 1-13, 2013.

[11] M. Ridolfi, S.Vandermeeren, and J. Defraye, "Experimental Evaluation of UWB Indoor Positioning for Sport Postures", Sensors, Vol. 18, No. 168, pp 1-20, 2018.

[12] S. Monica and G. Ferrari, "Accurate Indoor Localization with UWB Wireless Sensor Networks", In: Proc. of the 23rd International WETICE Conference, pp. 287-289, 2014.

[13] A. Yassin, Y. Nasser, and M. Awad, "Recent Advances in Indoor Localization: A Survey on Theoretical Approaches and Applications", University of New South Wales, Vol. 19, No. 2, pp. 1-21, 2016.

[14] A. Albaidhani, A. Morell, and J. Vicario, "Ranging in UWB using commercial radio modules: experimental validation and NLOS mitigation", In: Proc. of IEEE, IPIN Conference, pp. 1-7, 2016. 
[15] Decawave LTD. APS 006 Application notes, "Channel effects on communication range and time stamp accuracy in DW 1000 based system", Version 1.02, pp. 12-16, 2014.

[16] I. Brahmi, G. Abbruzzo, M. Walsh, H. Sedjelmaci, and B. Flynn, "A Fuzzy Logic Approach for Improving the Tracking Accuracy in Indoor Localization Applications", In: Proc. of IEEE -Wireless Days Conference, pp. 137144, 2018.

[17] D. Kim, G. Kwon, and J. Pyun, "NLOS Identification in UWB channel for Indoor Positioning", In: Proc. of IEEE Annual Consumer Communications and Networking Conference (CCNC), pp. 1-4, 2018.

[18] A. Alsudani, "NLOS Mitigation and Ranging Accuracy for Building Indoor Positioning System in UWB Using Commercial Radio Modules", In: Proc. of AIP Conf., pp. 1-8, 2018.

[19] B. You, X.Li, X. Zhao, and Y.Gao, "A Novel Robust Algorithm Attenuating Non-Line-ofSight Errors in Indoor Localization", In: Proc. of IEEE Conference (ICCSN) , pp. 1-6, 2015.
[20] Z. Xiao, H. Wen, and A. Markham, "NonLine-of-Sight Identification and mitigation using received signal strength", IEEE Transaction on Wireless Communications, Vol. 14, No. 3, pp. 1689-1702, 2015.

[21] Decawave Ltd. DW 1000 user manual, http//www.decawave.com, 2015.

[22] Y. Bai and D. Wang, "Fundamentals of Fuzzy Logic Control-Fuzzy Sets, Fuzzy Rules and Defuzzifications", Advanced Fuzzy Logic Technologies in Industrial Applications, pp. 1736.

[23] A. Albaidhani, A. Morell, and J. Vicario, "Anchor selection for UWB indoor positioning", Wiley, Anchor Selection for UWB Indoor Positioning, Vol. 30, No. 6, pp. 1-17, 2019.

[24] V. Barral, P. Casal, C. Escudero, and J. Naya, "Assessment of UWB Ranging Biasin Multipath Environments", In: Proc. of IEEE, IPIN Conference, pp. 1- 4, 2016. 


\section{Appendix}

Table 7. Measurement of distance where, $\mathrm{d}$, and Av express the real distance and the average of the distance bias respectively

\begin{tabular}{|c|c|c|c|c|c|c|c|c|c|c|c|c|}
\hline & \multicolumn{4}{|c|}{ LOS } & \multicolumn{4}{|c|}{ SNLOS } & \multicolumn{4}{|c|}{ HNLOS } \\
\hline $\begin{array}{l}d \\
(m)\end{array}$ & $\begin{array}{l}R S P \\
(\mathrm{dBm})\end{array}$ & $\begin{array}{c}\operatorname{dif} f \\
(\mathrm{~dB})\end{array}$ & $\begin{array}{l}\text { SNR } \\
\text { (dB) }\end{array}$ & $\begin{array}{l}\text { Bias } \\
(m)\end{array}$ & $\begin{array}{c}R S P \\
(\mathrm{dBm})\end{array}$ & $\begin{array}{c}\operatorname{dif} f \\
(\mathrm{~dB})\end{array}$ & $\begin{array}{l}\text { SNR } \\
(\mathrm{dB})\end{array}$ & $\begin{array}{l}\text { Bias } \\
\text { (m) }\end{array}$ & $\begin{array}{l}R S P \\
(\mathrm{dBm})\end{array}$ & $\begin{array}{l}\operatorname{dif} f \\
(\mathrm{~dB})\end{array}$ & $\begin{array}{r}\text { SNR } \\
(\mathrm{dB})\end{array}$ & $\begin{array}{c}\text { Bias } \\
(\mathrm{m})\end{array}$ \\
\hline 2 & -78.97 & -2.17 & 0.6 & 0.09 & - & - & - & - & - & - & - & - \\
\hline 4 & -79.22 & -4.23 & 0.3 & 0.08 & -79.8 & -4.24 & -5.3 & 0.19 & - & - & - & - \\
\hline 6 & -79.81 & -4.85 & -0.3 & 0.02 & -85.85 & -3.25 & -6.5 & 0.26 & - & - & - & - \\
\hline 8 & -79.83 & -3.27 & 0.28 & 0.05 & -90.66 & -5.41 & -11 & 0.27 & -87.3 & -17.89 & -7.3 & 1.33 \\
\hline 10 & -79.39 & -2.46 & 0.2 & 0.05 & -94.76 & -7.84 & -15 & 0.11 & -86.83 & -21.98 & -11 & 0.74 \\
\hline 12 & -79.72 & -1.36 & -0.3 & 0.01 & -96.60 & -8.23 & -17 & 0.10 & -91.38 & -17.29 & -11.8 & 0.58 \\
\hline 14 & -79.75 & -1.38 & -0.2 & -0.01 & -93.18 & -4.24 & -13 & 0.50 & -92.54 & -9.11 & -13.1 & 1.00 \\
\hline 16 & -79 & -2.33 & 0.00 & 0.08 & -91.07 & -6.01 & -11.6 & 0.35 & -89.42 & -12.56 & -9.6 & 0.94 \\
\hline 18 & -80.53 & -0.88 & -1.00 & 0.02 & -90.24 & -4.72 & -11 & 0.24 & -89.2 & -17.19 & -9.5 & 0.48 \\
\hline 20 & -80.78 & -2.70 & -1.3 & 0.70 & -88.81 & -5.18 & -9 & 0.50 & -89.70 & -16.49 & -9.3 & 0.69 \\
\hline 22 & -81.37 & -1.37 & -1.9 & 0.11 & - & - & - & - & - & - & - & - \\
\hline $\begin{array}{l}A v \\
\text { Bias }\end{array}$ & - & - & - & 0.05 & - & - & - & 0.3 & - & - & - & 0.82 \\
\hline
\end{tabular}

